\title{
Portable Centrifugal Microfluidic System for Diagnostics in Resource-Limited Settings
}

\author{
Christopher R. Phaneuf -Sandia National Laboratories, Victoria A. VanderNoot-Sandia National \\ Laboratories, and Chung-Yan Koh -Sandia National Laboratories
}

\begin{abstract}
The threats of disease outbreaks and exposure to biothreat agents, both accidental and intentional, demand fielddeployable technology capable of rapid, sensitive, and accurate diagnosis. In order to address these public health concerns, we present a portable centrifugal microfluidic platform and demonstrate sensitive detection protein antigens, host response antibodies, and nucleic acids down to single digit starting copies. The nucleic acid detection utilizes an isothermal amplification via loop-mediated isothermal amplification (LAMP). The platform, which is composed of a compact optical system for laser induced fluorescence (LIF) detection, a quiet brushless motor, and an efficient non-contact heater, offers an easy-to-use system capable of performing sensitive biodetection in a constrained-resource environment.
\end{abstract}

\section{INTRODUCTION}

We have previously reported on the development of a microfluidic system designed at Sandia National Laboratories to conduct and to develop novel immunoassays $[1,2]$. In order to further mature the technology and expand the capabilities of the platform, a heating system was incorporated to enable nucleic acids tests using techniques such as LAMP. Previous implementations of heated centrifugal systems in the literature typically suffer from complicated and unreliable approaches for power delivery such as the use of a slip ring for electrically interfacing with the rotating microfluidic device as required for traditional heating elements [3,4]. Slip rings have the potential to introduce greater complexity, significantly limit the available rotational speeds, and have finite lifetimes due to wearing down of the graphite or metal brushes. Other strategies have been published, such as induction heating [5], which offers the benefits of a non-contact solution but typically requires extensive circuitry and on-disc electrodes. These elements

This work was supported by the National Institute of Allergy and Infectious Diseases of the National Institutes of Health under Award Number R01AI098853. The content is solely the responsibility of the authors and does not necessarily represent the official views of the National Institutes of Health. Sandia National Laboratories is a multi-program laboratory managed and operated by Sandia Corporation, a wholly owned subsidiary of Lockheed Martin Corporation, for the U.S. Department of Energy's National Nuclear Security Administration under contract DEAC04- 94AL85000.

C.-Y. Koh is with Sandia National Laboratories, Livermore, CA 94551 USA (phone: 925-294-2845; fax: 925-294-3282; e-mail: ckoh@sandia.gov).

V.A. VanderNoot is with Sandia National Laboratories, Livermore, CA 94551 USA. (e-mail: vavande@sandia.gov).

C.R. Phaneuf is with Sandia National Laboratories, Livermore, CA 94551 USA. (e-mail: crphane@sandia.gov). increase cost and complexity of disc fabrication, decreasing the utility of the device as a fieldable platform for low resource settings. Infrared laser heating has been demonstrated but is only suitable for small targets and also contributes to increased disc complexity by requiring an embedded metal plate to achieve high temperatures via indirect heating of the sample [6]. Thermoelectric heating, commonly used for PCR thermocyclers, has been implemented but requires additional moving parts, such as a linear actuator [7], to bring the disc into contact with the heat source. The non-contact heating system presented here provides temporally stable and spatially uniform temperature control with a simple, reliable set of components.

\section{EXPERIMENTAL METHODS}

\section{A. Immunoassay detection}

Protein detection proceeds via a novel sedimentationbased microparticle pull-down through a density matrix. Microparticles are covalently decorated with target-specific antibodies to form the capture surface. These microparticles are then incubated with the sample in the presence of a fluorescently-labeled detection antibody for fifteen minutes. In the presence of the analyte of interest, the detection antibody and the capture antibody form an immunocomplex on the microparticle. This immunocomplex is able to pass through the density medium preloaded into a microfluidic channel. The particles are washed by Stokes' flow while passing through the density medium, separating bound and unbound reporter antibody. The particles are then concentrated at the tip of the channel at the periphery of the disc. The signal is read via laser-induced fluorescence; increased fluorescent signal is directly correlated with increased analyte concentration. If the analyte of interest is not present, the reporter antibody will be unable to form stable immunocomplexes on the surface of the microparticle. These unbound molecules will be separated from the microparticles during sedimentation and no signal will be seen at the tip of the channel. Similarly, serology assays can be performed on the platform. Rather than immobilizing a capture antibody, antigen of interest can be attached to the surface. These assays are then quantified with a fluorescently-labeled secondary antibody.

\section{B. Molecular diagnostics}

The platform is primarily composed of three subsystems: 1) optical system for LIF, 2) rotary control system, and 3) 
non-contact heating system. The optical system consists of standard elements, including a laser diode module, photomultiplier tube, and excitation and emission filters. The rotary control system is built around a brushless DC servomotor with an absolute encoder. Optical switches interact with markings on the disc for home positioning. The heating system is based on a custom carbon filament medium-wave infrared emitter mounted in a hinged enclosure in order to swivel the heater into position over the disc when ready for operation. While the disc rotates at 100 RPM, the heater is powered at $28 \mathrm{~W}$ to achieve uniform heating to $65^{\circ} \mathrm{C}$. These components, along with a power supply and control electronics, are housed in a Pelican case.

\section{RESULTS AND DISCUSSION}

\section{A. Molecular diagnostics}

The platform was first calibrated for open loop temperature control. Surface temperature was determined by thermal imaging and correlated with sample temperature within the microfluidic chambers with a purpose-built thermal probe. After temperature calibration, the platform was tested by amplifying a heat-killed $E$. coli $\mathrm{O} 157: \mathrm{H} 7$ target using a LAMP reaction with QUASR chemistry [8]. With a 10x serial dilution of the target DNA from 10,000 cells to $\sim 1$ cell, sets of $10 \mu \mathrm{L}$ reactions were run in triplicate for each template concentration along with a negative template control (NTC). The disc was heated to $65^{\circ} \mathrm{C}$ for $45 \mathrm{~min}$, allowed to cool, and fluorescence was measured. Successful detection over the range of dilutions was observed. Additionally, direct detection from clinical sample matrices has been demonstrated. The Bst polymerase used is highly resistant to some common PCR-inhibiting samples such as whole blood, urine, and saliva. In the $10 \mu \mathrm{L}$ format, clinical sample matrices were able to be analyzed without any sample preparation off device. A panel of enteric bacteria, including salmonella, campylobacter, shigella, and E. coli O157:H7, were tested in a variety of sample matrices to determine sensitivity and specificity. Detection of DNA was competitive with qPCR. Detection of RNA was typically demonstrated with a five-fold decrease in sensitivity. The reverse transcription step is known to be difficult in LAMP reactions and further work is ongoing.

\section{B. Immunoassay detection}

A corresponding set of immunoassays were developed to complement the nucleic acid detection tests. Assays for markers on the bacterial surface were developed. Additionally, assays for excreted protein toxins were also developed, such as shigatoxin. Limits of detection for the whole cell immunoassays were in the single cell range per assay. The limits of detection for the protein toxins were in the picogram per milliliter range. Sensitivity for the immunoassay in the microfluidic platform is enhanced for several reasons. The microparticles used exhibit very low autofluorescence in the wavelengths of interest, allowing for enhanced signal-to-noise with very low background noise. Additionally, using microparticles increases the effective surface area for binding the target protein analyte to the surface of the microparticle. For a typical assay, more than one hundred times the area of a 96- well plate is used for a single test. The microfluidic channels enhance reaction kinetics and shorten incubation times. The microfluidic channels additionally narrow to a point for detection, physically concentrating the signal. Finally, by utilizing a density medium to create a barrier between the microparticle capture surface and interfering compounds or unbound signal with stringent washing, the assay greatly decreases the potential for false positives while maintaining a simple onestep procedure for the end user.

As illustrated in Table 1, the platform is able to perform sensitive immunoassays directly from a clinically relevant sample matrix. The stability, specificity, and activity of the antibodies used for detection have a direct impact on the sensitivity of the assays.

TABLE I. LIMITS OF DETECTION AND QUANTIFICATION FOR VARIOUS BIOLOGICAL TOXINS OF PUBLIC HEALTH CONSEQUENCE.

\begin{tabular}{|c|c|c|}
\hline \multirow{2}{*}{ Toxin } & \multicolumn{2}{|c|}{ Figures of Merit } \\
\cline { 2 - 3 } & $\boldsymbol{L o D}(\mathbf{p g} / \mathbf{m L})$ & $\operatorname{LoQ}(\mathbf{p g} / \mathbf{m L})$ \\
\hline BoNT/A & $0.5^{\mathrm{a}}$ & 9 \\
\hline BoNT/B & 40 & 60 \\
\hline Ricin & 12 & 65 \\
\hline Shiga & 14 & 48 \\
\hline SEB & 130 & 280 \\
\hline
\end{tabular}

a. Values are calculated from an 8-point dose response curve fitted with a 4 parameter logistic in OriginPro. All measurements were taken on platform with toxin spiked into whole mouse blood.

\section{CONCLUSION}

We have demonstrated sensitive and specific detection of a variety of biological molecules on a centrifugal microfluidic platform. This platform, being portable and easy to use, may have potential for field deployment in resourceconstrained environments and enable laboratory-grade results without the laboratory. The ability to detect both nucleic acids and proteins on the same platform is somewhat unique in the point-of-care community.

\section{REFERENCES}

[1] U. Y. Schaff and G. J. Sommer, "Whole Blood Immunoassay Based on Centrifugal Bead Sedimentation," Clinical Chemistry, vol. 57, no. 5, pp. 753-761, May 2011.

[2] C.-Y. Koh, U. Y. Schaff, M. E. Piccini, L. H. Stanker, L. W. Cheng, E. Ravichandran, B.-R. Singh, G. J. Sommer, and A. K. Singh, "Centrifugal Microfluidic Platform for Ultrasensitive Detection of Botulinum Toxin," Anal. Chem., vol. 87, no. 2, pp. 922-928, Jan. 2015.

[3] R. Martinez-Duarte, R. A. G. Iii, K. Abi-Samra, and M. J. Madou, "The integration of 3D carbon-electrode dielectrophoresis on a CDlike centrifugal microfluidic platform," Lab Chip, vol. 10, no. 8, pp. 1030-1043, Apr. 2010.

[4] K. Abi-Samra, T.-H. Kim, D.-K. Park, N. Kim, J. Kim, H. Kim, Y.-K. Cho, and M. Madou, "Electrochemical velocimetry on centrifugal microfluidic platforms," Lab Chip, vol. 13, no. 16, pp. 3253-3260, Jul. 2013. 
[5] X. Chen, L. Song, B. Assadsangabi, J. Fang, M. S. Mohamed Ali, and K. Takahata, "Wirelessly addressable heater array for centrifugal microfluidics and Escherichia coli sterilization," in 2013 35th Annual International Conference of the IEEE Engineering in Medicine and Biology Society (EMBC), 2013, pp. 5505-5508.

[6] T.-H. Kim, J. Park, C.-J. Kim, and Y.-K. Cho, "Fully Integrated Labon- a-Disc for Nucleic Acid Analysis of Food-Borne Pathogens," Anal. Chem., vol. 86, no. 8, pp. 3841-3848, Apr. 2014.

[7] M. Amasia, M. Cozzens, and M. J. Madou, "Centrifugal microfluidic platform for rapid PCR amplification using integrated thermoelectric heating and ice-valving," Sensors and Actuators B: Chemical, vol. 161, no. 1, pp. 1191-1197, Jan. 2012.

[8] C. S. Ball, Y. K. Light, C.-Y. Koh, S. S. Wheeler, L. L. Coffey, and R. J. Meagher, "Quenching of Unincorporated Amplification Signal Reporters in Reverse-Transcription Loop-Mediated Isothermal Amplification Enabling Bright, Single-Step, Closed-Tube, and Multiplexed Detection of RNA Viruses," Analytical Chemistry, vol. 88, no. 7, pp. 3562-3568, Mar. 2016. 